\title{
Tra Storia e Mito: L'ossimoro Siciliano Secondo Gesualdo Bufalino
}

\author{
Flaviano Pisanelü
}

ABSTRACT: Definendosi un "siculo europeo", Bufalino, nella sua opera letteraria e poetica (in particolare L'amaro miele e Museo d'ombre) come nei suoi numerosi articoli giornalistici e interventi critici raccolti in volume (Cere perse, La luce e il lutto), elabora sonorità e significati che tentano di restituire le sfumature cromatiche e l'immagine a mosaico di una Sicilia che non è solo "metafora" - come ha avuto modo di scrivere Sciascia - di una civiltà mediterranea in continua trasformazione, ma anche uno spazio in cui, attraverso il dispositivo memoria, è possibile rivisitare un patrimonio storico-culturale, materiale e immateriale, capace di affermarsi come principio identitario in costante evoluzione. In questa prospettiva, lo spazio insulare siciliano diventa una fonte inesauribile di rappresentazioni e di autorappresentazioni sulle quali Bufalino sviluppa una personalissima poetica e un immaginario che restituiscono alla Sicilia la sua vocazione naturale di "isola-ponte" e di "isolaplurale" da sempre influenzata dal razionalismo europeo e da una sorta di magismo mitico che soffia con forza dalle rive meridionali del mare nostrum.

PAROLE CHIAVE: Bufalino; Sicilia; spazio mediterraneo; identità; frontiera; interculturalità.

* Université Paul-Valéry - Montpellier III 
RESUMO: Definindo-se um "siciliano europeu”, Gesualdo Bufalino, em sua obra literária e poética (especialmente em L'amaro miele e Museo d'ombre) assim como em seus inúmeros artigos jornalísticos e intervenções críticas reunidos em volume (Cere perse, La luce e il lutto), elabora sonoridades e significados que tentam devolver as nuances cromáticas e a imagem em formato de mosaico de uma Sicília que não é apenas "metáfora" - como Leonardo Sciascia escreveu-de uma civilização mediterrânea em contínua transformação, mas também um espaço em que, por meio do dispositivo da memória, é possível revisitar um patrimônio histórico e cultural, material e imaterial, capaz de se afirmar como principio identitário em constante evolução. Nessa perspectiva, o espaço insular siciliano se torna uma fonte inesgotável de representações e autorrepresentações nas quais Bufalino desenvolve uma poética personalíssima e um imaginário que devolvem à Sicília sua vocação natural de "ilha-ponte" e de "ilha-plural" desde sempre influenciada pelo racionalismo europeu e por uma espécie de magismo mítico que sopra com força das margens sulistas do mare nostrum.

PALAVRAS-CHAVE: Bufalino; Sicília; espaço mediterrâneo; identidade; fronteira; interculturalidade.

ABSTRACT: Defining himself as a "Sicilian-European", Bufalino, in his literary and poetic works (in particular his L'amaro miele and Museo d'ombre) as well as his numerous journalistic articles and reviews (in the collections Cere perse and La luce e il lutto), elaborates sonorities and significations which attempt to reinstate the chromatic nuances and the mosaic imagery of a Sicily which is not only a "metaphor" - as Sciascia often wrote - of a Mediterranean civilization in continual transformation, but also a space in which, by means of memory positioning, it is possible to revisit a heritage at once historical and cultural, material and immaterial, capable of affirming itself as an identifying principle in constant evolution. In this sense the insular Sicilian space becomes an inexhaustible fount of representations and self-representations from which Bufalino develops a highly personal poetic and 
an imaginative world which reestablishes Sicily in its natural vocation of "islandbridge" and "islands-plural" forever influenced by European rationalism and a sort of magic-mythic blown forcefully from the southern shores of mare nostrum. KEY-WORDS: Bufalino; Sicily; space Mediterranean; identity; border; interculturality. 
«La Sicilia non ha mai smesso di essere un grande ossimoro geografico e antropologico di lutto e luce, di lava e miele.»

(Bufalino, Cere perse)

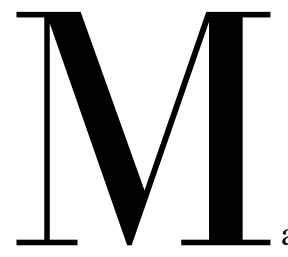

aria Corti, nell'introduzione all'edizione delle Opere di Gesualdo Bufalino (1996), scrive che in molti dei suoi testi letterari e saggistici l'autore esprime, attraverso il dispositivo dell'ossimoro, il sentimento d'appartenenza ad uno spazio insulare liberato e assediato, aperto e chiuso dalla doppia valenza geografica e simbolica del mare e, al contempo, la volontà di inscriversi all'interno di una radice culturale plurale e multipla che, sin dai tempi della Grecia classica, caratterizza lo spazio del Mediterraneo. Franco Cassano, riflettendo sul modello geopolitico elaborato nei secoli dalla cultura ellenica, torna sull'importanza del rapporto dialettico che si instaura tra mare e terra, il quale avrebbe peraltro favorito la comunicazione e gli scambi tra le diverse civiltà che hanno abitato e abitano ancora il bacino del Mediterraneo: 
Il rapporto tra le differenze (con le loro dinamiche complesse, conflittuali e spesso tragiche) è qui sin dall'inizio il problema. Questo mare ad un tempo esterno e interno, abitato e guadato, questo mare-confine produce un'interruzione del dominio dell'identità, costringe ad ospitare la scissione. Qui la terra con la sua ossessione per la fissità, la sicurezza e l'appropriazione urta sempre contro un limite, qui è stata da subito più difficile la confusione tra il governo di una città e il potere del proprietario. E questo grazie all'insinuarsi e all'interporsi del mare, alla sua capacità di custodire e collegare le differenze con la loro talvolta irresistibile resistenza a federarsi, che solo il grande nemico comune (l'impero persiano) riesce a smorzare. (CASSANO, 1996, p. 23-24).

Definendosi un "siculo europeo", Bufalino, nella sua opera letteraria e poetica (in particolare L'amaro miele e Museo d'ombre), come nei suoi numerosi articoli giornalistici e interventi critici raccolti in volume (Cere perse, La luce e il lutto), elabora sonorità e significati che tentano di restituire le sfumature cromatiche e l'immagine a mosaico di una Sicilia che non è solo "metafora" - come ha avuto modo di scrivere Sciascia - di una civiltà mediterranea in continua trasformazione, ma anche uno spazio in cui, attraverso il dispositivo memoria, è possibile rivisitare un patrimonio storico-culturale, materiale e immateriale, capace di affermarsi come principio identitario in costante evoluzione.

In questa prospettiva, lo spazio insulare siciliano diventa una fonte inesauribile di rappresentazioni e di autorappresentazioni sulle quali Bufalino sviluppa una personalissima poetica e un immaginario che restituiscono alla Sicilia la sua vocazione naturale di 'isolaponte' e di 'isola-plurale' da sempre influenzata dal razionalismo europeo, tipico della sponda settentrionale del Mediterraneo e tendente a fissare limiti geografici e identitari, e da una sorta di magismo mitico che, soffiando con forza dalle rive meridionali del mare nostrum, spinge alla scoperta o alla riscoperta, mai definitive, di una radice identitaria ancestrale condivisa.

Nell'eccesso di luce e d'ombra, di vita e di morte, che sono alla base della spontanea e spesso naturale teatralità del vivere siciliano, i confini spazio-temporali e identitari dell'isola sembrano al contempo imporsi e annullarsi fino a trascolorare in una scrittura che rende visibile l'invisibilità di una storia ancora tutta da rileggere e da decifrare. In uno dei suoi studi dedicati alla letteratura di autori delle isole del Mediterraneo, Margherita Marras spiega come la circolarità spazio-temporale, che contribuisce con forza all'elaborazione del mito insulare, sia spesso 
associata all'immagine di un'isola sulla quale la storia non riesce ad avere alcuna presa sul reale:

En ce qui concerne la circularité temporelle, les auteurs procèdent presque toujours à la mise en cuvre d'une dialectique qui voit le temps de l' " horloge » se superposer à celui du mythe, le temps de l'histoire se confronter et s'affronter au temps suspendu. (MARRAS, 2005, p. 126).

Questa dicotomia, originando una sorta di rapporto dialettico inesauribile tra la mobilità extra-insulare e una certa immobilità culturale dell'isola, legata spesso a valori e sistemi sociali ancestrali e arcaici, condiziona ed influenza la rappresentazione dello "spazio-tempo" costruita in molti testi letterari di autori siciliani, per esempio nei romanzi di Giuseppe Bonaviri o, in tempi più recenti, di Silvana Grasso.

Gesualdo Bufalino dedica allo spazio insulare natale, tormentato tra storia e mito, mobilità e immobilità, molte pagine della sua opera, nelle quali l'isola è al tempo stesso soggetto e oggetto delle rappresentazioni e delle autorappresentazioni più diverse. Nel testo Pro Sicilia, l'autore scrive:

Quel cielo e quel mare, miracolosamente resistenti agli insulti della chimica; i vulcani in fiamme, le miti colline; le pianure dove scorrono fiumi dal nome di miele; le leggende che fioriscono sulle labbra in un'aria di mito; le botteghe dove artigiani impareggiabili ripetono i venerandi gesti della fatica; le finestre fiorite di graste, dietro cui una ragazza bruna sorride; le chiese di pietra bionda, belle come creature di carne; le piazze dove ogni giorno il cartellone prevede una puntata nuova di quel teatro di pupi che è l'inesauribile vita; gli uomini, i milioni di uomini piccoli e scuri, dal cuore ospitale, benché così irto di sofismi e rovente di lave crudeli... (BUFALINO, 1996, p. 1139).

In questo breve passo, Bufalino esprime chiaramente il senso ossimorico della sua terra come in una sorta di iato infinito, fisico e interiore, che è capace di modificarne i contorni e spostarne incessantemente la frontiera. Appare subito l'immagine di una Sicilia iperreale e mitica, fissa e in movimento, amalgamata e divisa da elementi naturali (il cielo, il mare, il vulcano, la terra) che ne costituiscono la figura e l'ossatura, caratterizzata da un'evidente teatralità del quotidiano, 
dal gesto ripetuto dell' artigiano che, nel suo rito giornaliero, si fa custode di saperi antichi. A tutto questo si aggiunge la forza dell' epos che si traduce in leggenda, l'antropomorfizzazione di un patrimonio artistico, culturale e religioso irripetibile che difende dall'omologazione socioculturale contemporanea e, allo stesso tempo, ricorda la resistenza all'invasore sempre pronto a varcare le soglie del perimetro insulare. Notiamo, infine, il contrasto evidente nella tempra del siciliano tra il senso dell'ospitalità, dell'apertura allo "straniero" e il suo spinoso sistema culturale fatto di sofismi che hanno tuttavia permesso di difendere l'isola dai pericoli naturali e umani ai quali essa è da sempre esposta. La Sicilia intera sembra essere avviluppata da una "dolce violenza", una violenza di difesa che è andata costruendosi sulla nozione dell'eccesso, di cui anche Sciascia parla nel testo La Sicilia come metafora:

Chi sbarca a Palermo è subito preso da un'aria di violenza. Violenza di certe iscrizioni murali. Violenza di un cielo troppo azzurro quando è azzurro; troppo tormentato quando si prepara un temporale. Violenza soprattutto di secoli di sole e di eternità di polvere. Violenza dello scirocco, questo vento rosso venuto dall'Africa che vi stringe la testa in una morsa di fuoco mentre copre di sabbia $i$ tetti, le macchine, le strade. Ci si sente alla fine del mondo in Sicilia. Non appena si passa lo Stretto di Messina e il treno comincia a danzare sui binari divenuti all'improvviso troppo stretti, la velocità rallenta. (SCIASCIA, 1979, p. 6).

Lo Stretto di Messina, passaggio obbligato per chi arriva dal continente, è rappresentato da Bufalino, nel già citato articolo intitolato Pro Sicilia, come un luogo d'ombra e di pena, ma anche come uno spazio abitato da odori e colori di debordante vitalità:

È come se navigando tra Scilla e Cariddi, sul solco della nave due sirene affiorassero e vi tentassero con due lusinghe contrarie: una celeste, che parla di gelsomini d'Arabia, letizie di luna, spiagge simili a guancie dorate; l'altra scura, infera, con mezzogiorni ciechi a picco sulle trazzere e sangue che s'asciuga adagio ai piedi di un vecchio ulivo. (BUFALINO, 1996, p. 1135).

Lo spazio diviso tra ombra e luce, senso del mistero e reminiscenze mitiche dello Stretto è immerso in un'atmosfera doppio-multipla nella quale lo sguardo sembra assorbito e vinto da 
una foschia persistente e da vapori densi e incolori che l'Etna emette da tempi immemorabili. In questo testo, Bufalino ricorda alcuni versi scritti durante il periodo della sua adolescenza, nei quali egli fa riferimento ad uno dei suoi tanti passaggi e attraversamenti tra l'isola e il continente: "Sul traghetto di Messina / come due carabinieri / un angelo e un demonio / m'hanno preso sottobraccio, / all'orecchio destro e manco / m'hanno detto una parola...". Si tratta senz'altro di una parola separata, bifronte, di luce e d'ombra, aperta e chiusa alla storia: ad una storia che ha saputo nei secoli tagliare e ricucire, che ha ricordato e dimenticato culture e popoli, favole e leggende. Quest'ambivalenza della Sicilia evidenzia ancora una volta la sua doppia natura: l'una divina e angelica, l'altra solforosa e demoniaca. L'isola diventa così una sorta di terra-frontiera tra culture e identità che si ritrovano e si "ri-conoscono" all'interno di un unico spazio poroso e condiviso.

L'eccesso di luce costituisce per i siciliani quel palcoscenico sul quale più che vivere la vita, la si rappresenta. La luce accecante costituisce e delimita quasi lo spazio vitale in cui l'abitante dell'isola perpetua il suo gioco di accettazione/rifiuto della storia, del sogno, della quotidianità e della pena, della memoria e di un tempo che resta sempre ad un passo dal presente. In questo atteggiamento ludico fatto di continue "in-lusioni" e "de-lusioni”, la Sicilia ha saputo preservare le molteplici sfumature della sua identità di isola-ponte, nonostante la non sempre facile integrazione e metabolizzazione di culture e di lingue che l'hanno abitata e spesso dominata. ${ }^{1}$ D'altronde il fascino estetico delle architetture greche, arabo-normanne, ispaniche o barocche di molte città dell'isola nasconde effettivamente una storia a tratti dolorosa, fatta di ripetute invasioni e stagioni interminabili di sottomissione, da cui deriverebbe quello scetticismo attribuito, a torto o a ragione, al carattere siciliano. A questo proposito, Sciascia scrive:

Credo che un mondo di libertà e di giustizia può essere realizzato, anche se non in modo perfetto. Ma tutta la storia siciliana è una storia di disfatte: disfatte della ragione, disfatte degli uomini di ragione. La mia stessa storia è una storia di disfatte. O più modestamente di disillusioni. Donde lo scetticismo: che non è effettivamente accettazione della disfatta, ma margine di sicurezza, di elasticità che fa sì che la disfatta - già prevista, già ragionata - non divenga mai né definitiva né mortale. Lo scetticismo è salutare. È il miglior antidoto contro il fanatismo. (SCIASCIA, 1979, p. 45).

1 «Les excès constituent d'ailleurs la vertu de la Sicile et ils découlent bien sûr de la très compliquée et douloureuse histoire de l'île. La mer qui l'entoure de tous les côtés a permis aux Grecs et aux Carthaginois de débarquer sur l'île, aussi bien qu'aux Berbères et aux Normands, aux soldats lombards et aux avides barons de Charles d'Anjou, à l'armée de Charles Quint et aux Autrichiens, aux Piémontais et aux garibaldiens, avant que l'île ne soit rattachée au Royaume d'Italie soumis au pouvoir de la monarchie de Savoie.» (PISANELLI, 2005, p. 470). 
L'immagine sempre bipolare di un'identità costruita tra mito e realtà, l'esitazione tra l'esporsi e il difendersi dall'azione corrosiva della storia verrebbero, come Bufalino scrive nel testo L'isola plurale, dal pessimismo che il siciliano ha sempre nutrito nei confronti della ragione e della volontà:

Si tratta di un pessimismo della ragione, al quale quasi sempre si accompagna un pessimismo della volontà. Evidentemente la nostra ragione non è quella di Cartesio, ma quella di Gorgia, di Empedocle, di Pirandello. Sempre in bilico fra mito e sofisma, tra calcolo e demenza; sempre pronta a ribaltarsi nel suo contrario, allo stesso modo di un'immagine che si rifletta rovesciata nell'ironia di uno specchio. (BUFALINO, 1996, p. 1141);

A questo sentimento di paura e di pessimismo storico, di solitudine e d'isolamento, il siciliano oppone paradossalmente, come in un gioco di specchi asimmetrici, una tendenza eccessiva alla festa e all'euforia collettiva, soprattutto quando si confronta con il lutto e con la morte. In diversi testi raccolti nel volume La luce e il lutto - il cui titolo, costituendo uno spazio ossimorico, lascia già trasparire la molteplicità, la complessità e lo spirito contraddittorio dell'universo siciliano -, Bufalino si focalizza sulla relazione che si instaura tra l'eccesso di luce e l'importanza che la cultura dell'isola attribuisce al lutto. L'autore ricorda come in Sicilia le manifestazioni collettive legate al lutto siano capaci di esprimere un rapporto vivificante e prossimo tra l'individuo e la morte, al punto che la morte rappresenta quasi un momento di festa tragica capace di dar voce ad una sorta di passione collettiva. La morte sembra costituire il principale atto drammaturgico di una rappresentazione oscillante tra il sentimento del lutto e l'eccesso di luce:

[...] Così come l'isola tutta è una mischia di lutto e di luce. Dove è più nero il lutto, ivi è più flagrante la luce, e fa sembrare incredibile, inaccettabile la morte. Altrove la morte può forse giustificarsi come l'esito naturale d'ogni processo biologico; qui appare uno scandalo, un'invidia degli dei. (BUFALINO, 1996, p. 1141)

La morte in Sicilia si afferma come un puro scandalo, un paradosso, come un fatto quasi innaturale. È per questo motivo che il rito legato al lutto si trasforma in dispositivo capace di opporsi all'inaccettabile e di risanare l'azione sconvolgente e fatalistica della storia e della 
natura. Se la morte ossessiona l'uomo, bisogna che questi reagisca attraverso un atto eccessivo, totale, assoluto: l'eccesso della luce e la "messa in scena" (drammatizzazione della morte) riportano la situazione entro il territorio dell'accettabile, limitando così l'incursione feroce della storia nella quotidianità individuale e collettiva dell'isola.

Nel testo Palmina Enne Enne, sua morte, battesimo, esequie (BUFALINO, op. cit., p. 11501151), Bufalino torna sul legame che esiste in Sicilia tra la morte e l'euforia, relazione che ha permesso il fiorire di numerosi riti svolti in occasione del lutto e della sepoltura del defunto. In questo senso, la scena e la pena - di conseguenza il mimo e il rito - ritrovano nella morte il loro punto di incontro e di sintesi. Il mimo esprime, attraverso la forza del gesto, i parossismi della passione che alleviano il dolore interiore, mentre il rito collettivo canalizza lo slancio irrazionale della passione all'interno di un sistema comportamentale comune e condiviso che lo domina, lo epura e, per certi versi, lo neutralizza. La ritualità ha di conseguenza la funzione di risolvere lo scandalo della morte che è a sua volta "riparato" da un atto collettivo tradotto ed espresso sotto forma di liturgia, di sogno o di pura follia. Ancora una volta il "gioco" - inteso come ludo - e il sogno eccedono sulla ragione e sulla nozione diacronica tipica del tempo storico.

Questa tendenza a negare il flusso diacronico del tempo si riscontra in modo assai esplicito anche in certi usi verbali del dialetto siciliano che privilegia per esempio l'impiego del passato remoto quando si raccontano fatti o eventi piuttosto recenti. La nozione del tempo diventa nella lingua e nell'immaginario siciliani un vero e proprio paradosso: il passato recente e il presente sono quasi immunizzati e neutralizzati dal movimento storico grazie ad una memoria che preserva e custodisce congelando e mitizzando il flusso del tempo storico attraverso lo slittamento della narrazione al passato remoto. Il presente è in questo modo inscritto all'interno della dimensione atemporale del sogno, della visione, della ricordanza o della sfera del factum, del perfectum: tutto accade senza che niente accada realmente nel presente, tutto si pronuncia e si rivela nell'impossibilità di dire e di abitare fino in fondo il cambiamento. Questo 'presente' compiuto una volta per tutte è al contempo verità e menzogna, parola e silenzio, mistero imparlabile e progetto conclusus.

Nell'opera Museo d'ombre, Bufalino, rievocando antichi mestieri, luoghi scomparsi, antiche locuzioni dialettali e volti che hanno dato voce e corpo a personaggi diventati simbolo di una terra spesso alla deriva, fa viaggiare il lettore attraverso una Sicilia separata e scissa tra un qui e un li spazio-temporali in cui però l'ombra - intesa come traccia del visibile - resta custode di una memoria che è testimonianza, resistenza e prova inconfutabile di ciò che nel corso dei 
secoli ha fondato, trasformato, forgiato quell'identità siciliana e quell'immaginario che oggi si tende a sintetizzare con il termine di "sicilitudine". L'opera si apre con una breve prosa altamente poetica dedicata al lampionaio, ' $U$ Lampiunaru, ove ancora una volta l'autore fa riferimento alle nozioni di luce e d'ombra:

Copiava palesemente i gesti di un qualche barbuto Padreterno o stregone ' $u$ lampiunaru che all'imbrunire, appoggiata la scala a un fanale, si accingeva a sprigionarvi dentro, mediante un semplice zolfanello, i solenni miracoli della luce. Più misera vista all'alba: quando nelle piccole pensili case di vetro cominciavano a impallidire le fiamme, e lui sopravveniva di soppiatto e sotto il tocco molle della sua canna spegnimoccoli, come un sicario, ad una ad una le soffocava. (BUFALINO, op. cit., p. 160)

Nella figura del lampionaio ritroviamo un'immagine ossimorica, quella del dio-sicario, portatore e ladro di luce, responsabile della sua nascita e della sua morte. Nel gesto del "lampiunaru" si riassume l'effetto distruttore-conservatore che la storia esercita su uno spazio insulare che è al contempo reale, soggetto al fluire del tempo ("zolfanello", "piccole pensili case", "all'imbrunire [...] più misera vista all'alba") e luogo di un immaginario abitato dall'eternità ("Padreterno"), da "stregoni” e "miracoli della luce", dall'avvicendarsi di luce e lutto e, infine, dalla coscienza di una notte che resta lì, ad un passo dalla luce, forse entro la luce stessa del Meridione insulare. Il momento di sintesi - se di sintesi si può parlare - tra lo spazio reale e quello dell'immaginario è costituito nell'opera di Bufalino dalla "scrittura-memoria" che lega le due sfere e che rende attuale - nel suo doppio significato di atto e di gesto - ciò che sembra non avere più un corpo attraverso cui esprimersi. ${ }^{2}$ Si tratta di una "scrittura-memoria" che non solo recupera miticamente ombre, ma che agisce sul presente facendosi linguaggio e immagine, e, come sostiene lo stesso Bufalino, pedagogia e morale nelle quali 'ri-conoscersi':

Una civiltà è specialmente la ricchezza dei suoi mestieri. Ognuno dei quali nella propria cellula chiusa s'inventa mimiche, abbigliamenti, linguaggi, contegni, aneddoti di commozione o di scherzo, una pedagogia, una morale. [...] Più effimere ancora le attività vagabonde, esercitate all'aria aperta col consenso del

\footnotetext{
2 A questo proposito, Maria Corti sostiene che l'opera di Bufalino si costruisce essenzialmente su una memoria capace di produrre fantasmi, cioè immagini e figure che sono al contempo se stesse e rappresentazione di altro: «Nel primo caso, dice Aristotele, il fantasma è nell'anima al pari di un pensiero; nel secondo caso, che si chiarisce bene con una frase di Bergson, il fantasma si mette in contatto con la realtà passata e "se prolonge en action naissante", sicché a questo punto la memoria "ne nous représente plus notre passé, elle le joue”. È il processo che Bufalino insistentemente chiama 'sogno della memoria'. Ė proprio tale potere ludico della memoria che guida lo scrittore all'artificio dell'invenzione, a trasformare il ricordo in fiaba, in fantamemoria appunto» (CORTI. In BUFALINO, op. cit., p. XII-XIII).
} 
sole, della pioggia, del vento: mestieri da picaro; immagini, per un bambino che so, di invidiata felicità. (BUFALINO, op. cit., p. 159).

La sfiducia nella ragione geometrica, sistematica e dialettica, come anche la paura nei confronti della storia, tipiche della cosiddetta "sicilitudine", spingono il siciliano a non chiedersi mai - o quasi mai - le ragioni che producono un evento, un accadimento. Su tutto sembra dominare l'eccesso di luce, la tragedia corale del lutto, il rito collettivo che non rivelano, che non illuminano, che non esplicitano mai definitivamente il senso dell'evento per paura, per rabbia o forse solamente per salvaguardare quel mito imparlabile che alimenta da secoli la tendenza di quest' isola ad abbandonarsi alla rêverie o alla funzione affabulatoria della parola, dell'epos, del racconto, come è testimoniato dall'opera letteraria di tanti autori insulari, da Pirandello a Sciascia, da Brancati a Quasimodo, solo per citarne alcuni.

Attraverso l'eccesso di vita e di morte, la sottile coerenza della contraddizione, la mancanza di misura, il sentimento ludico nei confronti della realtà, la digressione infinita verso il tempo inesauribile del sogno, la Sicilia (e la sua letteratura) ha saputo preservare il suo ruolo di "isola-ponte" nei due sensi: come terra d'accoglienza e di partenza, come luogo di incontro e di separazione. Uno spazio ossimorico dunque, un'utopia intesa come "luogo-non luogo" nel quale potersi ritrovare solo rimanendo in cammino, nell'iter, nella distanza, nell'essere costantemente diversi da se stessi e dall'altro.

Questa è anche la Sicilia plurale e "utopica" vissuta, evocata e rappresentata dai tanti autori non italiani, e spesso non mediterranei, che in varie epoche sono approdati sull'isola: pensiamo alla Sicilia degli dèi raccontata da Johann Wolfgang Goethe, Lawrence Durrell, Bernard Berenson, Aléxis de Tocqueville; alla Sicilia dei "luoghi e delle immagini" visti dagli occhi e dalla penna di David Herbert Lawrence, Guy de Maupassant, Ibn Gubayr; o ancora la Sicilia dei mestieri, del lavoro, del gioco, della festa e del teatro che torna in molti scritti di Gaston Vuillier, Marguerite Yourcenar e Isaak Babel. A ciascuno la "sua" Sicilia, sulla quale proiettare dissidi, paure e utopie capaci di restituire sempre uno dei mille ritratti possibili di questa terra insulare, come si può facilmente evincere dalla lettura dei numerosi testi dedicati alla Sicilia e raccolti nel volume Cento Sicilie. Testimonianze per un ritratto, pubblicato nel 1993 e scritto a quattro mani da Gesualdo Bufalino e Nunzio Zago, attuale direttore scientifico della Fondazione Gesualdo Bufalino.

Ma l'ossimoro siciliano a cui Bufalino ha dato voce non si esaurisce nella mera nozione 
d'opposizione, del contrario, come anche Pasolini, fra gli altri, ha più volte spiegato. L'ossimoro è per Bufalino l'unica misura possibile capace di far coesistere gli opposti in una sorta di "sineciosi" e di coerenza che permettono di essere simile fra dissimili e dissimile fra simili, in modo da potersi ritrovare in un'utopia condivisa. Nell'opera Il Malpensante (1987), che si presenta come una raccolta di aforismi che non vogliono essere né precetti né assiomi, ma semplicemente abbozzi di ipotesi, impressioni, come in un antico almanacco, Bufalino scrive: "L'ossimoro non è una ridondanza ma una contrazione, non uno scialo ma un'economia." (BUFALINO, op. cit., p. 1045). L'ossimoro è dunque da intendersi paradossalmente come "contrazione", come spazio di senso simultaneo in cui convivono le divergenze e come forma di "economia", la cui etimologia (dal greco oikia + nomos) rinvia all'insieme delle norme che regolano il buon funzionamento della casa. Bufalino invita oggi il suo interlocutore a leggere da questa prospettiva il concetto di "sicilitudine" che torna con forza in molti suoi scritti.

Nella sua opera sempre in bilico tra poesia e prosa, nei suoi barocchismi espressivi intesi come forma di dissidenza e di resistenza ad un sentimento che l'autore stesso definisce attraverso un neologismo isolitudine, nei suoi scarti tematico-formali che toccano temi universali quali la memoria, la malattia, la morte e l'amore oscillanti tra metafisica ed esistenzialismo, tra ludi, cronaca, luce ed ombra, s'erge l'immagine di una Sicilia invasa e che invade, di una terra che è di per sé un palcoscenico sul quale si giocano paure, angosce e visioni, e che proprio grazie all'eccesso di luce arriva ad estendere i suoi confini geografici, culturali, sociali e letterari oltre quel mare che seduce, promettendo nuovi giochi, nuove vite, nuove e plurali verità. 


\section{Riferimenti bibliografici}

BUFALINO, G. Opere 1981-1988. Maria Corti e Francesca Caputo (Orgs.). 2 ed. Milano: Bompiani, 1996.

BUFALINO, G.; ZAGO, N. (Orgs.). Cento Sicilie. Testimonianze per un ritratto. Milano: Bompiani, $1993^{1}, 2008^{2}$.

CASSANO, F. Il pensiero meridiano. Roma-Bari: Laterza, 1996.

MARRAS, M. L'insularité entre imaginaire et réalité chez les romanciers sardes et siciliens

contemporains. In : TRABELSI, M. (Org.). L’insularité. Clermont-Ferrand: Presses Universitaires Blaise Pascal, 2005, p. 122-133.

PISANELLI, F. La Sicile au miroir: Leonardo Sciascia et Gesualdo Bufalino. In : TRABELSI, M. (org.). L’insularité. Clermont-Ferrand: Presses Universitaires Blaise Pascal, 2005, p. 467-488..

SCIASCIA, L. La Sicilia come metafora. Milano: Mondadori, 1979. 